\title{
All-optical radio-frequency modulation of Anderson-localized modes
}

\author{
G. Arregui, ${ }^{1,2}$ D. Navarro-Urrios, ${ }^{1}$ N. Kehagias, ${ }^{1}$ C. M. Sotomayor Torres,${ }^{1,3}$ and P. D. García ${ }^{1, *}$ \\ ${ }^{1}$ Catalan Institute of Nanoscience and Nanotechnology (ICN2), CSIC and The Barcelona Institute of Science and Technology, \\ Campus UAB, Bellaterra, 08193 Barcelona, Spain \\ ${ }^{2}$ Departament de Física, Universitat Autonoma de Barcelona, 08193 Bellaterra, Spain \\ ${ }^{3}$ ICREA - Institució Catalana de Recerca i Estudis Avançats, 08010 Barcelona, Spain
}

(Received 11 September 2018; revised manuscript received 25 October 2018; published 16 November 2018)

\begin{abstract}
All-optical modulation of light relies on exploiting intrinsic material nonlinearities [V. R. Almeida et al., Nature 431, 1081 (2004)]. However, this optical control is rather challenging due to the weak dependence of the refractive index and absorption coefficients on the concentration of free carriers in standard semiconductors [R. A. Soref and B. R. Bennett, Proc. SPIE 704, 32 (1987)]. To overcome this limitation, resonant structures with high spatial and spectral confinement are carefully designed to enhance the stored electromagnetic energy, thereby requiring lower excitation power to achieve significant nonlinear effects [K. Nozaki et al., Nat. Photonics 4, 477 (2010)]. Small mode-volume and high-quality $(Q)$-factor cavities also offer an efficient coherent control of the light field and the targeted optical properties. Here, we report on optical resonances reaching $Q \sim 10^{5}$ induced by disorder on photonic/phononic-crystal waveguides. At relatively low excitation powers (below $1 \mathrm{~mW}$ ), these cavities exhibit nonlinear effects leading to periodic (up to $\sim 35 \mathrm{MHz}$ ) oscillations of their resonant wavelength. Our system represents a test bed to study the interplay between structural complexity and material nonlinearities and their impact on localization phenomena and introduces a different functionality to the toolset of disordered photonics.
\end{abstract}

DOI: 10.1103/PhysRevB.98.180202

A successful strategy to achieve efficient optical confinement consists of introducing controlled point or line defects in otherwise regular dielectric lattices [1-3]. This has applications in both the classical and the quantum phenomena [4] such as slow light [5], efficient single-photon sources [6], nanolasing [7], optomechanical coupling [8], and even enhanced interaction between light and single atoms [9]. A bottleneck of this strategy relies on the extreme sensitivity of particularly high (as designed) $Q$-factor cavities $\left(Q \sim 10^{6}\right)$ to uncontrolled imperfections appearing during the fabrication process $[10,11]$. A less typical strategy to confine light consists of exploiting such imperfections [12]. Small spatial fluctuations of the order of few $0.001 a$ in the position of the lattice building blocks, where $a$ is the typical lattice parameter, give rise to strong multiple scattering which results in efficient optical confinement by recurrent interference with quality factors [13] reaching $Q \sim 10^{6}$, thus competing in performance with engineered defects while being inherently robust against disorder. Here, we exploit material nonlinearities to modulate these type of disorder-induced optical modes on a photoniccrystal waveguide which also allows for strong confinement of mechanical motion. Figure 1(a) displays a scheme of a shamrock-crystal waveguide, where the building block-the shamrock -is obtained by overlapping three ellipsoids rotated by $2 \pi / 3$ with respect to each other as detailed in Ref. [14] and in the Supplemental Material [15]. This structure is similar to a standard photonic-crystal waveguide [5] where the replacement of holes by shamrocks allows accommodating,

*http://www.icn.cat/ p2n/; david.garcia@icn2.cat simultaneously, a waveguide for THz-frequency photons and $\mathrm{GHz}$-frequency phonons for a lattice parameter $a=500 \mathrm{~nm}$. We fabricate our structures in silicon where material nonlinearities result [16] in an excess of free carriers and a significant structural heating under high optical excitation. As sketched in Figs. 1(b) and 1(c), these two processes become linked to each other while inducing an opposed dispersive effect on the refractive index of the structure, which eventually leads to closed stable trajectories in phase space and the periodic modulation of disorder-induced cavities at high radio frequency.

The optical modes induced by disorder in a shamrockcrystal waveguide, as the one shown in Fig. 2(a), appear around the slow-light region of the dispersion relation [13], in our case $\sim 1500 \mathrm{~nm}$ for $a=500 \mathrm{~nm}$. These modes are revealed in the transmitted light through a tapered-fiber loop $[8,17]$ when tuning a diode laser across this spectral region, as plotted in Fig. 2(b). When the fiber is placed in close proximity to the waveguide, as sketched in the inset of Fig. 2(b), the dips in transmission correspond to disorder-induced or Anderson-localized optical modes along the waveguide evanescently coupled to the fiber loop. These modes appear as peaks in the reflected signal measured with a 50/50 fiber beam splitter in the fiber input, as shown in Fig. 2(c). The band [18] of Anderson-localized modes can be frequency tuned by scaling the full in-plane structure by a factor $\Gamma$, as shown in Fig. 2(c), although the spectral position of individual modes within this band is uncertain due to the complex nature of the system. These modes present a rather broad distribution of $Q$ factors-plotted in Fig. 2(e)-with values in the range $1 \times 10^{3}<Q<1.5 \times 10^{5}$ and a mean 

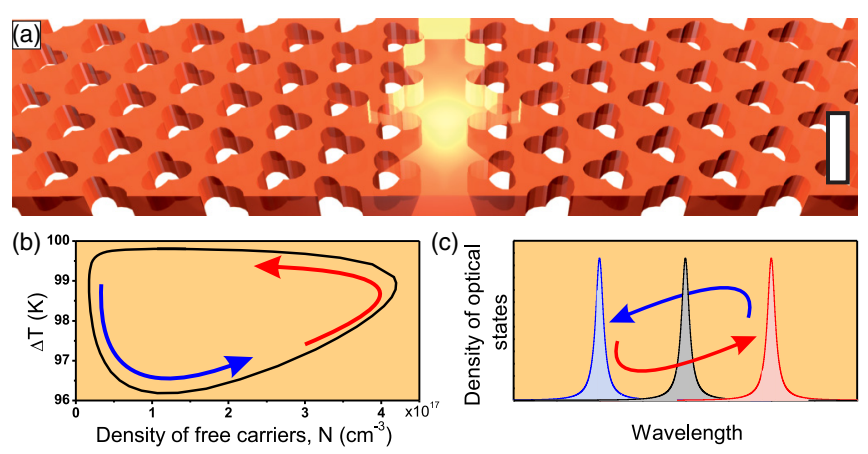

FIG. 1. Optical nonlinearity in a shamrock-crystal waveguide. (a) Illustration of a shamrock-crystal waveguide where the building block, the shamrock, is formed by overlapping three ellipsoids, rotated by $2 \pi / 3$ with respect to each other (details in Ref. [15]). The residual imperfection due to the fabrication process induces optical confinement along the waveguide. The scale bar is $500 \mathrm{~nm}$. (b) A limit cycle dynamic solution to the differential equations describing the nonlinear optical system. Here, the structural heating $\Delta T$ and the density of free carriers $N$ are linked to each other through the number of photons in the cavity, leading to a stable closed trajectory in phase space. (c) The dynamic variables of the system, $\Delta T$ and $N$, have an opposite dispersive effect on the refractive index of the structure which induces a temporal modulation of the disorder-induced mode. Here, the red and blue arrows represent the displacement along the dynamic solution in phase space and the induced spectral redshift or blueshift of the localized modes, respectively. value of $Q \sim 1.6 \times 10^{4}$. By comparing these values with the ones calculated with a fully three-dimensional Bloch mode expansion technique [19], we can estimate the amount of fabrication imperfection as $\sigma=0.006 a$, which is very similar to previous estimations of the tolerance of the fabrication process [20]. Here, we map all the possible different sources of intrinsic disorder to zero-mean Gaussian random displacements in the shamrock positions with standard deviation $\sigma$.

By analyzing the $Q$-factor distribution plotted in Fig. 2(e), we can extract very relevant information of the Andersonlocalized modes in our structure. The intrinsic values of this log-normal distribution are mainly determined by in-plane finite-size effects along the waveguide [21,22], i.e., by the average extension of the localized modes, $\xi$, compared to the total length of the system, $L=150 \mu \mathrm{m}$. Further loss mechanisms such as material absorption or out-of-plane leakage, which we quantify with the waveguide loss length $\ell$, may reduce these values limiting the maximum $Q$ factors achievable. In this picture, the ratio $\xi / L$ determines the log-normal values, while the ratio $\ell / L$ may impose a truncation to the distribution (see details in the Supplemental Material [15]). By fitting the measured distributions with a truncated log normal, we obtain $\xi / L=0.045 \pm 0.015$ and $\ell / L>10^{4}$. These values open the possibility for light-matter strong coupling induced by disorder, as calculated in Ref. [23]. The spectral fluctuations plotted in Figs. 2(b) and 2(c) can be further used to obtain the dimensionless conductance $g$ which quantifies the confinement induced by disorder in our structures. The smaller its value, the stronger is the confinement. Figure 2(e) plots the probability
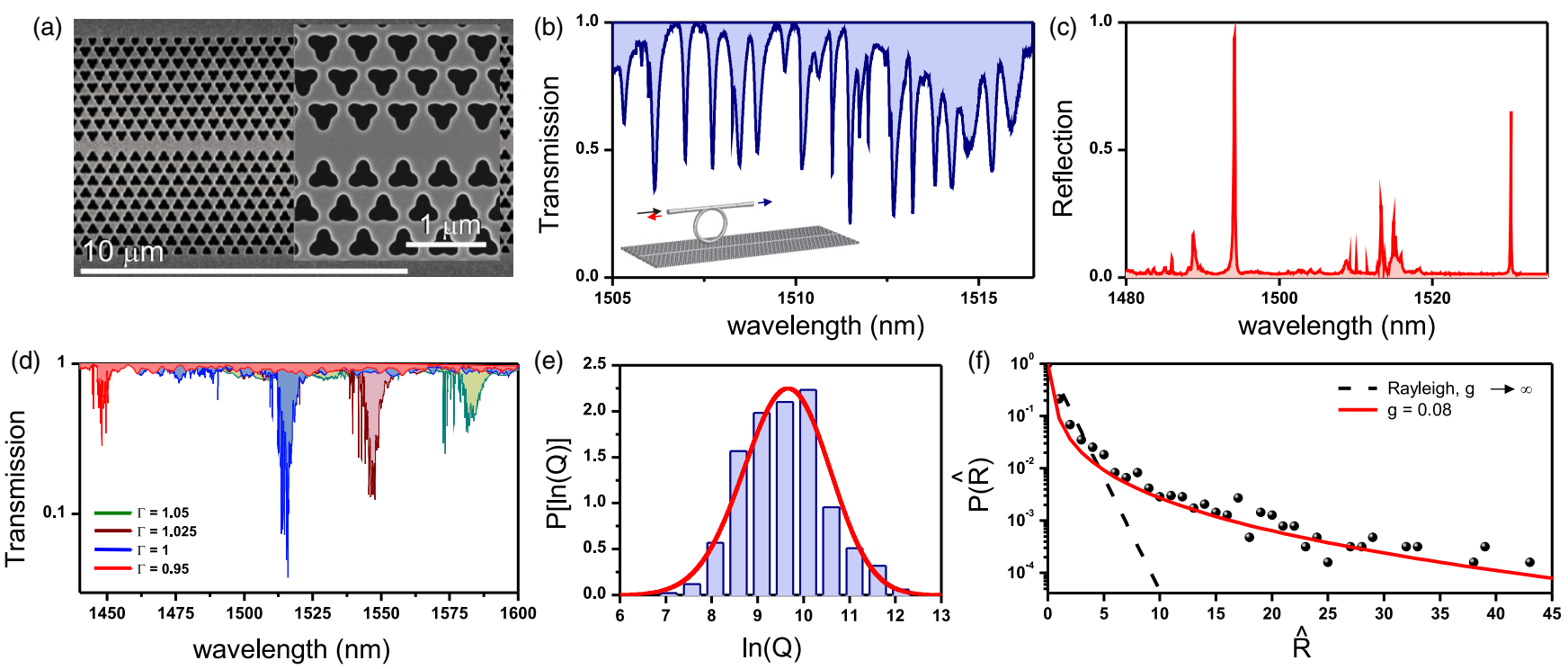

FIG. 2. Disorder-induced localization in shamrock-crystal waveguides. The optical modes of the structure are probed through a taperedfiber loop which is carefully placed in close proximity to the waveguide. (a) Scanning electron micrograph of a silicon shamrock-crystal waveguide obtained by etching two shamrock crystals with opposed symmetry and lattice constant $a=500 \mathrm{~nm}$. (b) Light from a tunable diode laser is transmitted through the fiber and couples evanescently with the localized modes of the structure, revealed as dips in transmission and peaks in reflection (c). (d) These localized modes appear in the slow-light regime of the waveguide and are redshifted when the entire in-plane nanostructure is scaled by a factor $\Gamma$. The broad features shown in the spectra correspond to propagating modes of along waveguide. (e) Histogram of the experimental $Q$ factors observed along the waveguide. The red curve plots the calculated distribution with parameters $\xi / L=0.045 \pm 0.015$ and $\ell / L>10^{5}$, where $\xi$ is the localization length, $\ell$ is the loss length, and $L=150 \mu \mathrm{m}$ is the total length of the waveguide (more details in Ref. [15]). (f) Reflectance probability distribution where $\hat{R}$ is the measured reflectance normalized to its average $\langle R\rangle$. The solid line represents the best fit to the theory of Ref. [24] with the dimensionless conductance $g$ as the single fitting parameter. 
distribution of the reflectance spectra collected by placing the fiber loop at different positions along the waveguide. We do this at the lowest input power $(5 \mu \mathrm{W})$ to rule out any effect of material nonlinearities. The tail of the distribution reveals the presence of a few but very bright peaks when compared to the background which is the fingerprint for Anderson localization. Fitting this distribution with the theory developed in Ref. [24] yields a dimensionless conductance of $g=0.08 \pm 0.01$ as a single fitting parameter, which is lower than in previous experiments $[12,25]$. To confirm this value, we calculate the Thouless number, i.e., the ratio between the average mode linewidth and mode spacing [26]. Calculated in reflection, this value $(0.06 \pm 0.02)$ is in agreement with the dimensionless conductance, although it differs from the value when calculated in transmission $(0.30 \pm 0.02)$. As shown in Figs. 2(b) and $2(\mathrm{c})$, the number of confined modes detected in reflection is lower, which reduces the collected statistics and gives rise to a larger average-mode spacing. This is due to the low power injected in the tapered fiber to avoid nonlinear effects which is mostly coupled evanescently to the confined modes. The small portion reflected is further slightly attenuated by the fiber coupler used in reflection configuration. However, the more conservative value of the Thouless number estimated in transmission still describes a waveguide well in the localized regime.

Large electromagnetic energy stored in a small volume results in a highly nonlinear behavior [27]. The resonant recirculation of weak excitations within an Anderson-localized mode is proportional to the input power and to the ratio $Q / V$. To estimate their typical mode profile and mode volume, we calculate the eigenmodes of a shamrock-crystal waveguide with the same structural parameters of the fabricated structures using a commercial finite-element solver. The total length of the simulated waveguide is $100 a$ and both sides of the structure are terminated with reflectionless absorbers in order to mimic an open system. The calculated waveguide is perturbed by a zero-mean Gaussian disorder in the position of the shamrocks with standard deviation $\sigma=0.006 a$ to reproduce the effect of the fabrication process (details in Ref. [15]). A calculated interference pattern along the waveguide is shown in Fig. 3(a) for a wavelength $\lambda_{c}=1512.95 \mathrm{~nm}$, revealing a typical Anderson-localized mode with a volume $V=1.2\left(\lambda_{c} / n\right)^{3}$. Here, $\lambda_{c}$ is the cavity wavelength in the linear regime-the cold wavelength - and $n$ is the material refractive index. These mode profiles and mode volumes are comparable to the ones of a carefully designed heterostructure-cavity mode [28]. As the intracavity optical-field intensity is enhanced in proportion to $Q / V$, a very low input power triggers nonlinear effects in the shamrock-crystal waveguide. We confirm this by increasing the input power of the excitation laser in our experiment. The transmission spectrum through the fiber-loop evanescently coupled to an Anderson-localized mode shows a typical sawtooth-shaped transmission bistability at an input power typically around $1 \mathrm{~mW}$, as plotted in Fig. 3(b). This power is slightly high when compared to other nanostructures where only confined modes are allowed [29]. The coexistence of confined with propagating modes in our waveguidesfeatured by the broad dips in the transmission spectra plotted in Fig. 2(d) - may open leaky channels to the coupled light, thus increasing the power required to achieve the nonlinear behavior. Finally, sweeping the excitation laser wavelength at higher powers drags the resonant wavelength to higher values, broadening the range of the hysteresis loop.

The main nonlinear process in standard silicon resonators in the telecom spectral range is two-photon absorption which generates free carriers [16]. This population eventually decays, generating phonons in the lattice, thus heating the structure. In addition, the induced free-carrier population absorbs additional photons which leads to further structural heating. In addition, these two parameters are linked, leading to complex dynamics of the resonant wavelength which can be described by a system of nonlinear coupled rate equations [30] (details in Ref. [15]). This phenomenon has been observed in different resonant photonic nanostructures such as microdisks [30], photonic crystals [31], or optomechanical crystals [29], but it is still unexplored in the context of disordered photonics. By measuring the oscillatory components of the transmitted light with a fast photodetector while sweeping the laser wavelength $\lambda_{L}$, it is possible to unravel the complex dynamics of a particular Anderson-localized mode with a cold cavity wavelength of $\lambda_{c}=1510.3 \mathrm{~nm}$ and $Q=6 \times 10^{4}$. Figure $3(\mathrm{c})$ plots this radio-frequency modulation starting with the laser blue detuned with respect to the Anderson mode. For low input laser wavelengths, the resonant wavelength presents a stable temperature-dominated and time-independent redshift. Increasing $\lambda_{L}$ beyond $1510.5 \mathrm{~nm}$, which corresponds to the intracavity photon threshold value [30] of $n_{0, \text { th }} \sim 1.35 \times 10^{5}$, leads to a periodic modulation of the intracavity mode wavelength [32,33], revealed as a clear frequency comb with increasing spacing. To understand the dynamics of this optical modulation, Fig. 3(d) sketches different frames of the period of the Anderson-localized mode oscillation around the laser wavelength marked as a black solid line. Figures 3(d)(1) $3(d)(3)$ plot the first half period of the oscillation when the cavity mode is progressively blueshifted due to a slow temperature recovery and a fast free-carrier population buildup, as sketched in Fig. 1(b). Far from resonance, at position (1), the transmission through the fiber at $\lambda_{\mathrm{L}}$ is maximum due to the poor coupling to the cavity mode. When the cavity is tuned on resonance with the laser-position (2) - the transmission through the fiber drops to the minimum due to the evanescent coupling of the transmitted light to the Andersonlocalized cavity. The oscillation still continues to the maximum blueshift at position (3), for which the effect of heating of the sample overcomes the effect of the excess of free carriers and redshifts the localized mode back to its initial position.

The full oscillation cycle is detected by time-resolving the transmission through the fiber at a fixed $\lambda_{L}$ with a fast oscilloscope. As shown in Figs. 3(e) and 3(f), the period decreases from $53.7 \mathrm{~ns}$ at $\lambda_{L}=1510.5 \mathrm{~nm}$ to $30.3 \mathrm{~ns}$ at $\lambda_{L}=1510.9 \mathrm{~nm}$. The fastest modulation happens at $\lambda_{L}=$ $1510.9 \mathrm{~nm}$, after which the period decreases until the oscillation is completely lost and the resonant wavelength of the mode returns to its initial value at $\lambda_{c}=1510.3 \mathrm{~nm}$. The complex dynamics shown here are due to an intricate interdependence of all the different physical parameters involved in the nonlinear rate equations which are developed in detail in Ref. [15]. Beyond the temporal modulation of the cavity, the material nonlinearities have an effect in the Andersonlocalization process. The optical nonlinearity has two main 

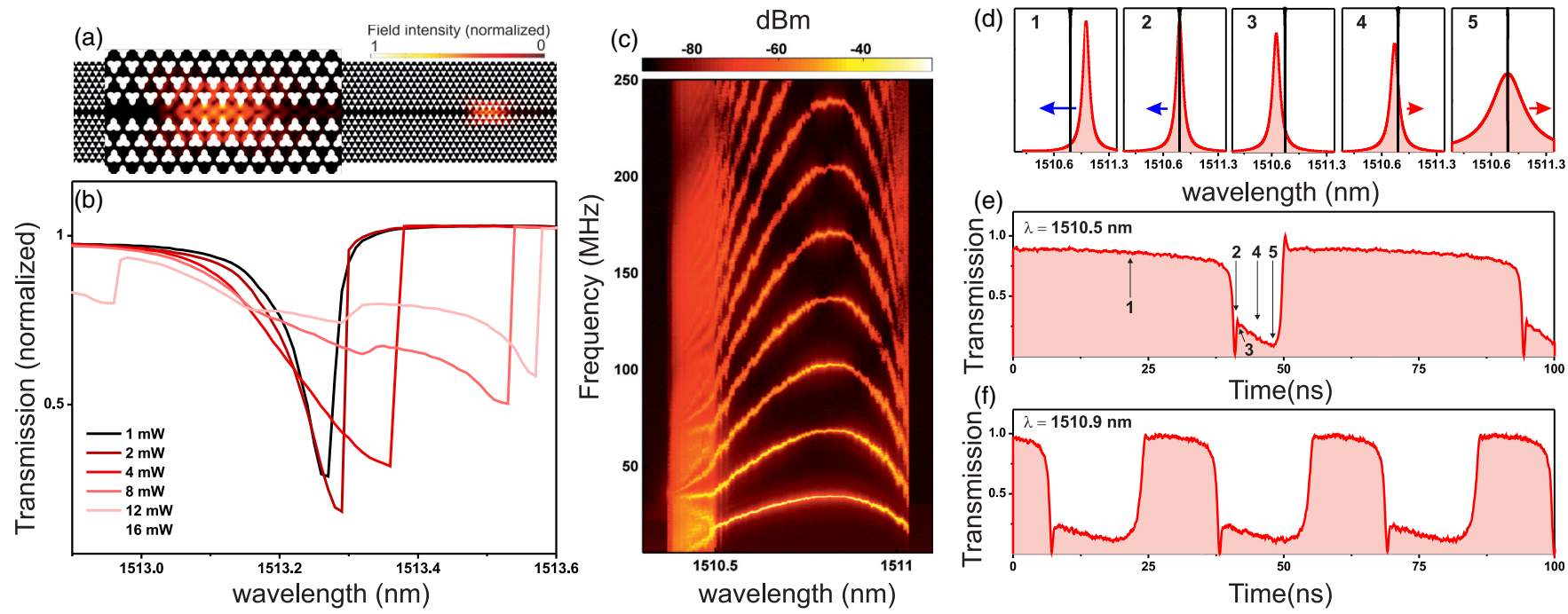

FIG. 3. Nonlinear modulation of Anderson-localized modes. (a) Light field intensity of a typical eigenmode of a shamrock-crystal waveguide with a lattice constant $a=500 \mathrm{~nm}$ (other parameters detailed in Ref. [15]), calculated around the cutoff frequency of the ideal slow-light waveguide mode. The position of the shamrocks is randomized normally with a standard deviation of $\sigma=0.006 a$ mimicking the effect due to the fabrication process. (b) Normalized transmission spectra of an Anderson-localized optical mode measured upon increasing the input power, which shows a typical sawtooth-shaped transmission bistability, caused by the material nonlinearities. (c) Color contour plot of the radio-frequency power spectral density at an input power of $16 \mathrm{~mW}$ measured while scanning the input laser wavelength $\lambda_{L}$ through an Anderson-localized mode with a cold wavelength at $\lambda_{c}=1510.3 \mathrm{~nm}$. (d) Sketch of the fast modulation cycle of an Anderson-localized mode around $\lambda_{L}$ marked with a solid-blue line. (e) and (f) Time-resolved transmission measured with an input laser wavelength at $\lambda_{L}=1510.5 \mathrm{~nm}$ and $\lambda_{L}=1510.9 \mathrm{~nm}$, respectively.

effects on the $Q$ factor of the Anderson-localized modes. First, the material two-photon absorption induces a dissipation mechanism which increases the optical leakage reducing the $Q$ factor of the modes. This has been observed in previous experiments with resonant nonlinearity in standard siliconphotonic structures $[16,29]$. However, the nonlinear process induces an additional dephasing mechanism in the interference process which leads to Anderson localization [34]. This decoherence mechanism further reduces the $Q$ factor and it is expected to fully destroy the localization effect in an infinite system [35]. As sketched in Fig. 3(d), the $Q$ factor of the Anderson-localized mode is reduced from position (2) to position (5). This fast modulation of the $Q$ factor explains why the two resonant conditions shown in Figs. 3(e) and 3(f) do not drop to the same value, a feature that cannot be explained by solely considering the dissipation induced by two-photon absorption and free-carrier-induced absorption. The multiple scattering process adds additional complexity to this picture. When few modes overlap spectrally and spatially, a complex collective behavior is expected in the dynamics of the system due to their interaction. The spectral shift induced by the material nonlinearity is strongly mode dependent which allows tuning different modes on resonance. This spatial and spectral mode overlap should result in complex temporal transmission traces that cannot be explained with a single-cavity model. However, we do not observe these interaction effects in our experiment. We attribute this to the suppression of mode interaction in the localization regime, even between modes that have significant spatial and frequency overlap, as predicted in Ref. [36] and confirmed by experiments on multimode lasing in the Anderson-localized regime [37].
In summary, we explore here the rich physics resulting from the complex interaction between multiple scattering and material optical nonlinearities. We exploit these nonlinearities to modulate resonant modes induced by disorder in the radiofrequency range. This all-optical modulation of disorderinduced modes add an extra functionality to the toolset of light-matter interaction mediated by disorder. Disordered photonics [38] offer an alternative and efficient platform for quantum electrodynamics [39] or lasing [37] which are inherently robust against disorder. These functionalities, in combination with the fast optical modulation shown here, allow all-optical data processing granted by imperfection rather than careful engineering. While the fundamental limits of engineered optical nanocavities are well known, the limits of this approach are still to be explored, however, the performance can be improved even further by inducing long-range disorder correlations [40]. Although not shown here, the shamrock nanostructure offers the possibility to couple the electromagnetic field with confined mechanical modes in the GHz-frequency range and to explore optomechanical effects in complex media.

This work was supported by the Spanish Ministerio de Ciencia, Innovación y Universidades (MICINN) via the Severo Ochoa Program (Grant No. SEV-2013-0295) and the project PHENTOM (Fis 2015-70862-P), as well as by the CERCA Programme/Generalitat de Catalunya, and by the European Commission in the form of the H2020 FET Open Project PHENOMEN (Grant No. 713450). G.A. is supported by a BIST Ph.D. Fellowship, and P.D.G. and D.N.-U. gratefully acknowledge support through the Ramon y Cajal fellowships No. RYC-2015-18124 and No. RYC-2014-15392, respectively. 


\section{APPENDIX: MATERIALS AND METHODS}

\section{Sample design and fabrication}

The shamrock-crystal waveguides were fabricated with standard silicon-on-insulator wafers with a top silicon layer thickness of $250 \mathrm{~nm}$ and a $2-\mu \mathrm{m}$-thick buried oxide sacrificial layer. The fabrication of the structure is based on an electron beam, direct-writing process performed on a coated 100-nm polymethyl-methacrylate resist film. The final shamrockcrystal waveguides are obtained by reactive-ion etching and isotropic vapor etching using hydrofluoride acid solutions. The total refractive index of the structure is $n=3.4$. A batch of samples was fabricated with a lattice constant $a=500 \mathrm{~nm}$, and a range of scaling factors $\Gamma=[0.95,1.00,1.025,1.05]$ and Gaussian disorder in the position of the shamrocks with standard deviations $\sigma=[0,0.1 a, 0.2 a, 0.3 a]$.

\section{Experimental setup and optical characterization}

A tapered-fiber loop with a diameter of $\sim 30 \mu \mathrm{m}$ is placed nearly parallel to the shamrock-crystal waveguide to excite its localized transverse electric (TE)-like optical modes. A diode laser tunable within $1400 \mathrm{~nm}<\lambda<1600 \mathrm{~nm}$ and a spectral resolution of $1 \mathrm{pm}$ is coupled to the fiber input and the transmitted light is detected at the output using two strategies. To measure the transmission spectrum, the light is sent directly to a slow photodetector while the reflected light is measured by coupling the photodetector to a 50/50 fiber beam splitter in the fiber input. The radio-frequency modulation of the transmitted light is measured with an InGaAs fast photodetector with a bandwidth of $12 \mathrm{GHz}$. The radio-frequency voltage is connected to the $50-\Omega$ input impedance of a signal analyzer with a bandwidth of $13.5 \mathrm{GHz}$. The transmitted light is time resolved with a 4-GHz oscilloscope. The whole setup operates at atmospheric conditions of temperature and pressure (see extended detailed information in Ref. [15]).

We measure a total of 25 input-output reflectance and transmittance curves by varying the position of the taperedfiber loop along the shamrock-crystal waveguide at the lowest input power of $0.2 \mu \mathrm{W}$. The loaded quality factor $Q_{\text {load }}$ is extracted by fitting the transmission dips with a Lorentzian function. From this experimental $Q_{\text {load }}$, we extract the intrinsic $Q$ factor as $Q_{\text {load }}=2 \mathrm{Q} /\left(1 \pm \sqrt{T_{\min }}\right)$, where $T_{\min }$ refers to the normalized transmission on resonance with the cavity [41]. The full $Q$-distribution data set is plotted in Fig. 2(d).

The reflectance probability distribution $P(\widehat{R})$ plotted in Fig. 2(e) is measured by collecting the reflectance intensity $R_{x, \lambda}$ at different positions of the waveguide $x$ while scanning the laser wavelength $\lambda$. Finally, $\widehat{R}$ is obtained at each position by subtracting the background and normalizing the resulting spectrum by the value averaged over the spectral range $1505-1520 \mathrm{~nm}$. Ergodicity on position and wavelength is assumed to obtain the final histogram plotted in Fig. 2(e).
[1] V. R. Almeida, C. A. Barrios, R. R. Panepucci, and M. Lipson, Nature (London) 431, 1081 (2004).

[2] R. A. Soref and B. R. Bennett, Proc. SPIE 704, 32 (1987).

[3] K. Nozaki, T. Tanabe, A. Shinya, S. Matsuo, T. Sato, H. Taniyama, and M. Notomi, Nat. Photonics 4, 477 (2010).

[4] P. Lodahl, S. Mahmoodian, and S. Stobbe, Rev. Mod. Phys. 87, 347 (2015).

[5] T. Baba, Nat. Photonics 2, 465 (2008).

[6] M. Arcari, I. Söllner, A. Javadi, S. Lindskov Hansen, S. Mahmoodian, J. Liu, H. Thyrrestrup, E. H. Lee, J. D. Song, S. Stobbe, and P. Lodahl, Phys. Rev. Lett. 113, 093603 (2014).

[7] S. Noda, Science 314, 260 (2006).

[8] M. Eichenfield, J. Chan, R. M. Camacho, K. J. Vahala, and O. Painter, Nature (London) 462, 78 (2009).

[9] A. Goban, C. L. Hung, S. P. Yu, J. D. Hood, J. A. Muniz, J. H. Lee, M. J. Martin, A. C. McClung, K. S. Choi, D. E. Chang, O. Painter, and H. J. Kimble, Nat. Commun. 5, 3808 (2014).

[10] D. Gerace and L. C. Andreani, Opt. Lett. 29, 1897 (2004).

[11] M. Minkov and V. Savona, Sci. Rep. 4, 5124 (2014).

[12] P. D. García and P. Lodahl, Ann. Phys. 529, 1600351 (2017).

[13] J. Topolancik, B. Ilic, and F. Vollmer, Phys. Rev. Lett. 99, 253901 (2007).

[14] I. Söllner, L. Midolo, and P. Lodahl, Phys. Rev. Lett. 116, 234301 (2016).

[15] See Supplemental Material at http://link.aps.org/supplemental/ 10.1103/PhysRevB.98.180202 for a detailed description of the structures (design and fabrication), and full characterization and derivation of the nonlinear equations describing the system, which includes Refs. [16,42-60].
[16] P. E. Barclay, K. Srinivasan, and O. Painter, Opt. Express 13, 801 (2005).

[17] M. W. Lee, C. Grillet, C. G. Poulton, C. Monat, C. L. C. Smith, E. Mägi, D. Freeman, S. Madden, B. Luther-Davies, and B. J. Eggleton, Opt. Express 16, 13800 (2008).

[18] I. M. Lifshitz, Adv. Phys. 13, 483 (1964).

[19] J. P. Vasco and S. Hughes, Phys. Rev. B 95, 224202 (2017)

[20] P. D. García, A. Javadi, H. Thyrrestrup, and P. Lodahl, Appl. Phys. Lett. 102, 031101 (2013).

[21] S. Smolka, H. Thyrrestrup, L. Sapienza, T. B. Lehmann, K. R. Rix, L. S. Froufe-Pérez, P. D. García, and P. Lodahl, New J. Phys. 13, 063044 (2011).

[22] V. Savona, Phys. Rev. B 83, 085301 (2011).

[23] H. Thyrrestrup, S. Smolka, L. Sapienza, and P. Lodahl, Phys. Rev. Lett. 108, 113901 (2012).

[24] M. C. W. van Rossum and T. M. Nieuwenhuizen, Rev. Mod. Phys. 71, 313 (1999).

[25] H. Hu, A. Strybulevych, J. H. Page, S. E. Skipetrov, and B. A. van Tiggelen, Nat. Phys. 4, 945 (2008).

[26] D. J. Thouless, Phys. Rev. Lett. 39, 1167 (1977).

[27] K. J. Vahala, Nature (London) 424, 839 (2003).

[28] B. S. Song, S. Noda, T. Asano, and Y. Akahane, Nature (London) 4, 207 (2005).

[29] D. Navarro-Urrios, N. E. Capuj, J. Gomis-Bresco, F. Alzina, A. Pitanti, A. Griol, A. Martínez, and C. M. Sotomayor Torres, Sci. Rep. 5, 15733 (2015).

[30] T. J. Johnson, M. Borselli, and Painter, Opt. Express 14, 817 (2006).

[31] J. Yang, T. Gu, J. Zheng, M. Yu, G. Q. Lo, D. L. Kwong, and C. W. Wong, Appl. Phys. Lett. 104, 061104 (2014). 
[32] S. Chen, L. Zhang, Y. Fei, and T. Cao, Opt. Express 20, 7454 (2012).

[33] D. Navarro-Urrios, N. E. Capuj, M. F. Colombano, P. D. García, M. Sledzinska, F. Alzina, A. Griol, A. Martínez, and C. M. Sotomayor-Torres, Nat. Commun. 8, 14965 (2017).

[34] I. V. Shadrivov, K. Y. Bliokh, Y. P. Bliokh, V. Freilikher, and Y. S. Kivshar, Phys. Rev. Lett. 104, 123902 (2010).

[35] N. Cherroret, J. Phys.: Condens. Matter 29, 024002 (2017).

[36] P. Stano and P. Jacquod, Nat. Photonics 7, 66 (2013).

[37] J. Liu, P. D. García, S. Ek, N. Gregersen, T. Suhr, M. Schubert, J. Mørk, S. Stobbe, and P. Lodahl, Nat. Nanotechnol. 9, 285 (2014).

[38] D. S. Wiersma, Nat. Photonics 7, 188 (2013).

[39] L. Sapienza, H. Thyrrestrup, S. Stobbe, P. D. García, S. Smolka, and P. Lodahl, Science 327, 1352 (2010).

[40] J. P. Vasco and S. Hughes, arXiv:1804.07854.

[41] M. Borselli, T. Johnson, and O. Painter, Opt. Express 13, 1515 (2005).

[42] M. Minkov, Numerical study and optimization of photonic crystals, Ph.D. dissertation, EPFL (Lausanne), 2016.

[43] M. Qiu, Appl. Phys. Lett. 81, 1163 (2002).

[44] P. D. García, G. Kiršanskè, A. Javadi, S. Stobbe, and P. Lodahl, Phys. Rev. B 96, 144201 (2017).

[45] T. Kottos, J. Phys. A: Math. Gen. 38, 10761 (2005).

[46] S. E. Pour Faez, Universal wave phenomena in multiple scattering media, Ph.D. dissertation, van der Waals-Zeeman Institute, 2011.
[47] F. A. Pinheiro, Phys. Rev. A 78, 023812 (2008).

[48] H. T. Nielsen, Quantum electrodynamics in photonic crystal waveguides, Ph.D. dissertation, Technical University of Denmark (DTU), 2011.

[49] P. Gregory, Bayesian Logical Data Analysis for the Physical Sciences - A Comparative Approach with Mathematica Support (Cambridge University Press, Cambridge, U.K., 2005).

[50] P. Sheng, Introduction to Wave Scattering, Localization, and Mesoscopic Phenomena (Academic Press, San Diego, 1995).

[51] E. Abrahams, P. W. Anderson, D. C. Licciardello, and T. V. Ramakrishnan, Phys. Rev. Lett. 42, 673 (1979).

[52] A. A. Chabanov, M. Stoytchev, and A. Z. Genack, Nature (London) 404, 850 (2000).

[53] A. W. Snyder and J. D. Love, Optical Waveguide Theory (Chapman and Hall, London, 1983).

[54] N. Cazier, X. Checoury, L.-D. Haret, and P. Boucaud, Opt. Express 21, 13626 (2013).

[55] W. H. Pernice, M. Li, and H. X. Tang, Opt. Express 18, 18438 (2010).

[56] S. Sze, Physics of Semiconductor Devices (Wiley, New York, 1981).

[57] M. Dinu, F. Quochi, and H. Garcia, Appl. Phys. Lett. 82, 2954 (2003).

[58] R. A. Soref and B. R. Bennett, IEEE J. Quantum Electron. 23, 123 (1987).

[59] T. Tanabe, H. Sumikura, H. Taniyama, A. Shinya, and M. Notomi, Appl. Phys. Lett. 96, 101103 (2010).

[60] G. Cocorullo and I. Rendina, IEE Electron Lett. 28, 83 (1992). 